\title{
Insights into the mechanism of hydroxyl radical mediated oxidations of 2- aminopurine: A Computational and sonochemical product analysis study
}

\author{
Kavanal P. Prasanthkumar, ${ }^{\# *}$ Manoj P. Rayaroth ${ }^{\S}$ and Juan R. Alvarez-Idaboy ${ }^{*}$
}

\#Post Graduate and Research Department of Chemistry, Maharaja's College, Ernakulam, Kerala, 682011, India.

${ }^{\$}$ School of Environmental Sciences, Mahatma Gandhi University, Kottayam, Kerala, 686560, India.

§Departamento de Física y Química Teórica, Facultad de Química, Universidad Nacional Autónoma de México, México D. F., 04510, México.

\section{Supporting Information}

\section{Contents}

1. Figure S1: Spin density plots of primary radicals

2. Table S1: The retention time (RT), $\mathrm{m} / \mathrm{z}$ values of TPs and their corresponding fragments

3. Figure S2: CID induced mass spectrum of $2 \mathrm{AP}$

4. Figure S3: CID induced mass spectrum of TP9

5. Figure S4: CID induced mass spectrum of TP4

6. Figure S5: CID induced mass spectrum of TP8

7. Figure S6: CID induced mass spectrum of TP14

8. Scheme S1 to Scheme S10: Assigned mass fragmentation patterns for 10 transformation products (TP1, TP2, TP3, TP5, TP6, TP7, TP10, TP11, TP12 and TP13) formed in hydroxyl radical reaction with 2AP. 


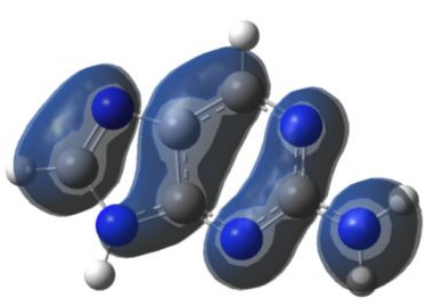

2AP॰+

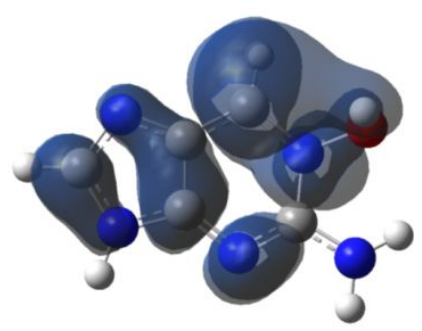

2AP-N10H•

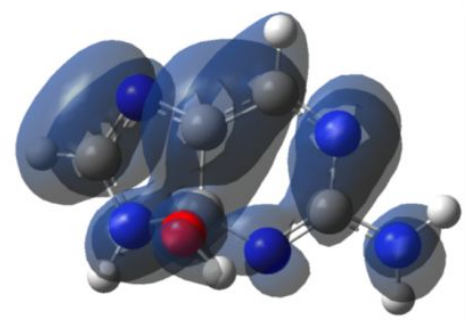

2AP-C4OH•

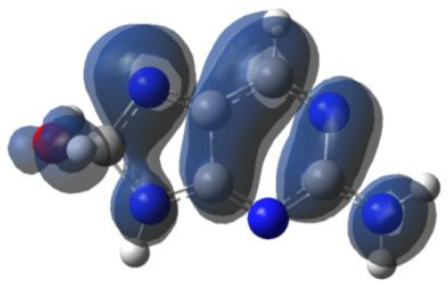

2AP-C8OH•

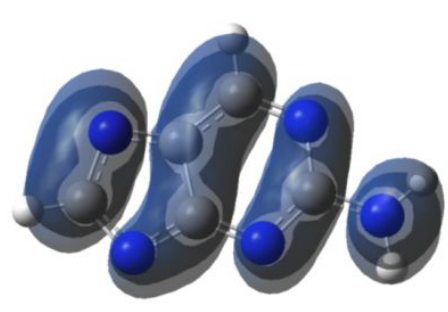

2AP-N9•

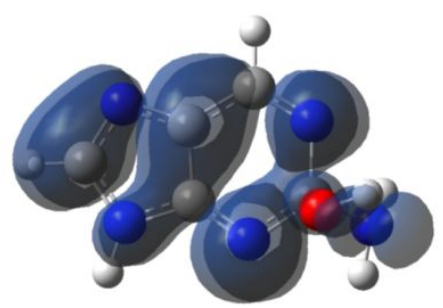

2AP-C2OH•

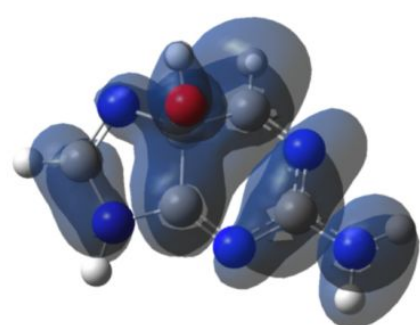

2AP-C5OH•

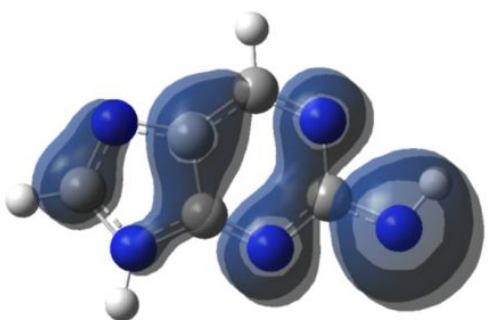

2AP-N10•

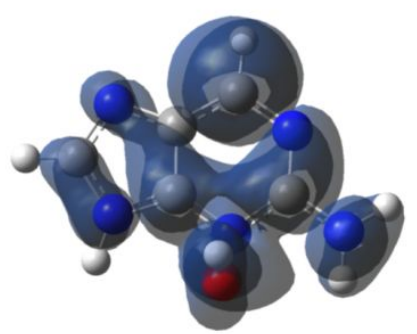

2AP-N3OH・

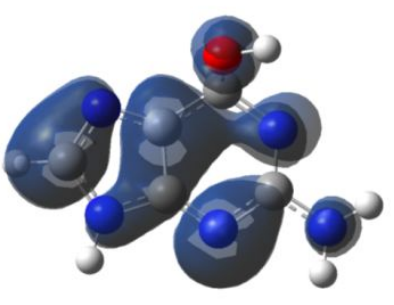

2AP-C6OH•

Figure S1: Spin density plots of primary radicals 
Table 2 Retention time (RT), Elemental Composition (EC) of $[\mathrm{M}+\mathrm{H}]^{+}$ions and $\mathrm{m} / \mathrm{z}$ values of 2AP \& TPs and their fragments. Boldface value in each row represents the base peak $\mathrm{m} / \mathrm{z}$ observed in the respective mass spectrum.

\begin{tabular}{|c|c|c|c|}
\hline \multirow{2}{*}{$\begin{array}{l}\text { Molecular } \\
\text { identity }\end{array}$} & \multirow{2}{*}{$\begin{array}{l}\mathrm{RT} \\
(\mathrm{min})\end{array}$} & \multicolumn{2}{|c|}{$\mathrm{m} / \mathrm{z}$ value: Experimental (Calculated, Error in $\mathrm{ppm}$ ) } \\
\hline & & $\begin{array}{l}\text { Parent }[\mathrm{M}+\mathrm{H}]^{+} \\
\text {ion }\end{array}$ & Fragment ions \\
\hline TP1 & 2.97 & $\begin{array}{l}161.0675 \\
(161.0670,3.10) \\
\text { EC: } \mathrm{C}_{4} \mathrm{H}_{9} \mathrm{~N}_{4} \mathrm{O}_{3}^{+}\end{array}$ & 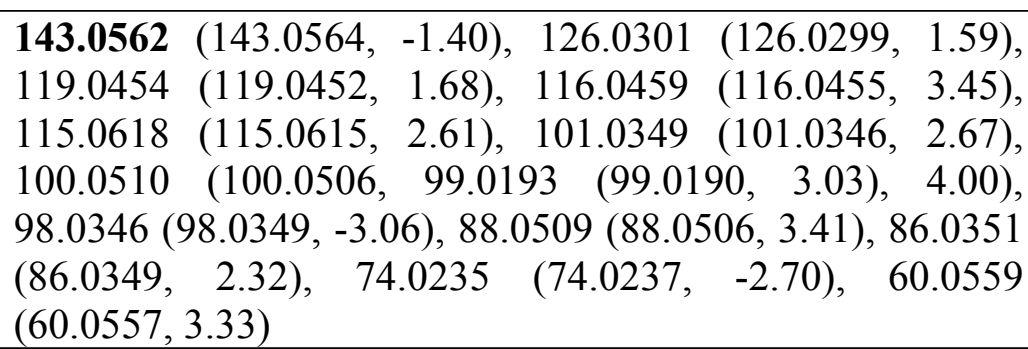 \\
\hline TP2 & 3.02 & $\begin{array}{l}168.0519 \\
(168.0519,1.79) \\
\text { EC: } \mathrm{C}_{5} \mathrm{H}_{6} \mathrm{~N}_{5} \mathrm{O}_{2}^{+}\end{array}$ & 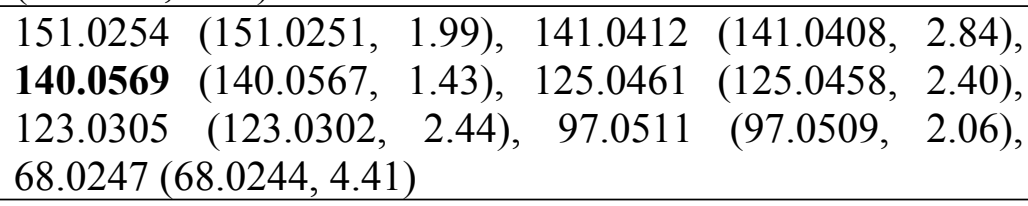 \\
\hline TP3 & 3.07 & $\begin{array}{l}204.0734 \\
(204.0728,2.94) \\
\text { EC: } \mathrm{C}_{5} \mathrm{H}_{10} \mathrm{~N}_{5} \mathrm{O}_{4}^{+}\end{array}$ & $\begin{array}{llllll}186.0622 & (186.0622, & 0.00), & 169.0363 & (169.0357, & 3.55), \\
153.0414 & (153.0408, & 3.92), & 144.0407 & (144.0404, & 2.08), \\
142.0252 & (142.0248, & 2.82), & 141.0411 & (141.0408, & 2.13), \\
126.0304 & (126.0299, & 3.97), & 114.0301 & (114.0299, & 1.75), \\
\mathbf{1 1 3 . 0 4 6 2} & (113.0458,3.54), 86.0352 & (86.0349,3.49) & \end{array}$ \\
\hline TP4 & 3.23 & $\begin{array}{l}154.0727 \\
(154.0724,1.95) \\
\text { EC: } \mathrm{C}_{5} \mathrm{H}_{8} \mathrm{~N}_{5} \mathrm{O}^{+} \\
\end{array}$ & 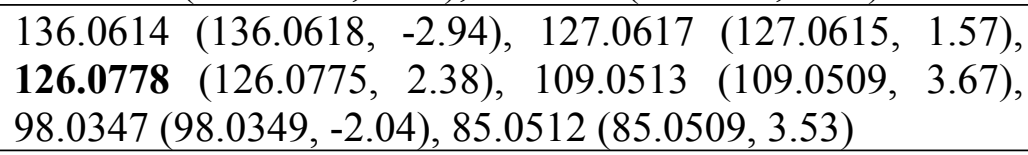 \\
\hline TP5 & 3.43 & $\begin{array}{l}156.0513 \\
(156.0516,-1.92) \\
\text { EC: } \mathrm{C}_{4} \mathrm{H}_{6} \mathrm{~N}_{5} \mathrm{O}_{2}^{+}\end{array}$ & $\begin{array}{l}139.0253(139.0251,1.44), 114.0300(114.0299,0.88), \\
113.0461 \quad(113.0458,2.65), 97.0035 \quad(97.0033,2.06), \\
85.0510(85.0509,1.18), 71.0243(71.0240,4.22), 68.0247 \\
(68.0244,4.41)\end{array}$ \\
\hline TP6 & 3.48 & $\begin{array}{l}174.0625 \\
(174.0622,1.72) \\
\text { EC: } \mathrm{C}_{4} \mathrm{H}_{8} \mathrm{~N}_{5} \mathrm{O}_{3}^{+}\end{array}$ & 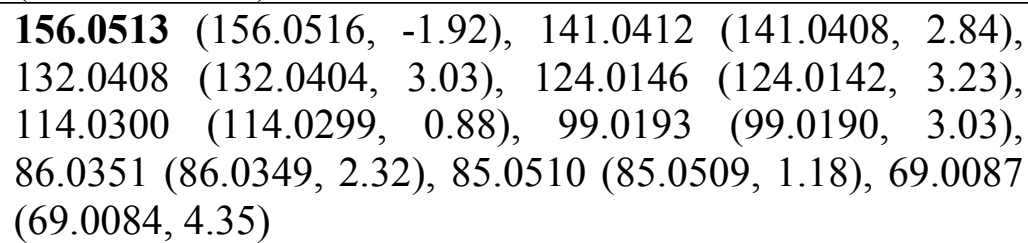 \\
\hline TP7 & 3.70 & $\begin{array}{l}129.0300 \\
(129.0295,3.88) \\
\text { EC: } \mathrm{C}_{4} \mathrm{H}_{5} \mathrm{~N}_{2} \mathrm{O}_{3}^{+} \\
\end{array}$ & $\begin{array}{l}87.0193(87.0190,3.45), 86.0236(86.0236,-1.16), 69.9926 \\
(69.9924,2.86), 68.9974(68.9972,2.90)\end{array}$ \\
\hline TP8 & 4.11 & $\begin{array}{l}152.0571 \\
(152.0567,2.63) \\
\text { EC: } \mathrm{C}_{5} \mathrm{H}_{6} \mathrm{~N}_{5} \mathrm{O}^{+}\end{array}$ & 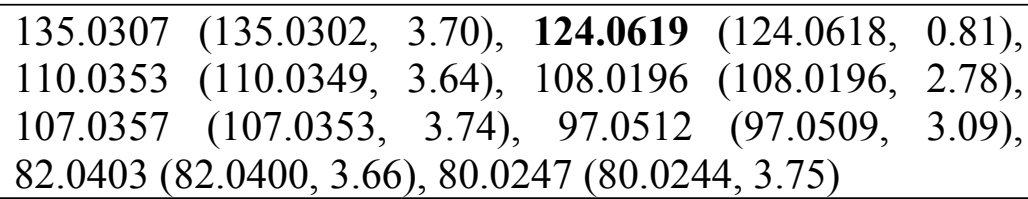 \\
\hline TP9 & 4.42 & $\begin{array}{l}152.0572 \\
(152.0567,3.29) \\
\text { EC: } \mathrm{C}_{5} \mathrm{H}_{6} \mathrm{~N}_{5} \mathrm{O}^{+}\end{array}$ & 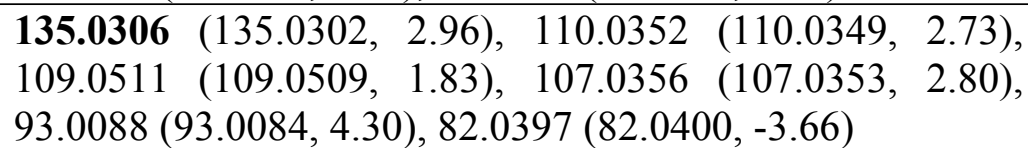 \\
\hline 2AP & 4.46 & $\begin{array}{l}\mathbf{1 3 6 . 0 6 1 9} \\
(136.0618,0.73)\end{array}$ & $\begin{array}{l}119.0356(119.0353,2.52), 109.0510(109.0509,0.92), \\
94.0403(94.0400,3.19), 92.0247(92.0244,3.26), 82.0403\end{array}$ \\
\hline
\end{tabular}




\begin{tabular}{|c|c|c|c|}
\hline & & $\mathrm{EC}: \mathrm{C}_{5} \mathrm{H}_{6} \mathrm{~N}_{5}{ }^{+}$ & $\begin{array}{l}(82.0400,3.66), 67.0293(67.0291,2.98), \\
65.0135,3.08), 55.0292(55.0291,1.82)\end{array}$ \\
\hline ТP10 & 5.11 & $\begin{array}{l}137.0463 \\
(137.0458,3.65) \\
\mathrm{EC}: \mathrm{C}_{5} \mathrm{H}_{5} \mathrm{~N}_{4} \mathrm{O}^{+}\end{array}$ & $\begin{array}{l}119.0349(119.0353,-3.36), 110.0352(110.0349,2.73), \\
94.0397(94.0400,-3.19), 92.0245(92.0244,1.09), 82.0403 \\
(82.0400,3.66), 67.0293(67.0291,2.98), 53.0137(53.0135, \\
3.77)\end{array}$ \\
\hline TP11 & 5.67 & $\begin{array}{l}171.0515 \\
(171.0513,1.17) \\
\mathrm{EC}: \mathrm{C}_{5} \mathrm{H}_{7} \mathrm{~N}_{4} \mathrm{O}_{3}{ }^{+}\end{array}$ & 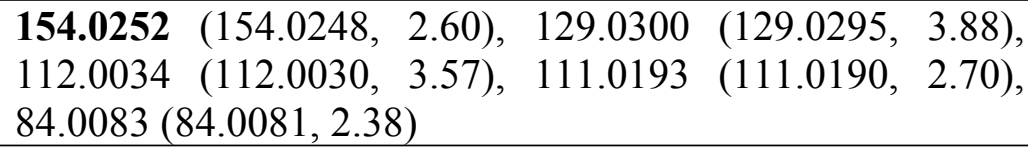 \\
\hline TP12 & 5.71 & $\begin{array}{l}194.1253 \\
(194.1248,2.58) \\
\text { EC: } \mathrm{C}_{5} \mathrm{H}_{16} \mathrm{~N}_{5} \mathrm{O}_{3}{ }^{+}\end{array}$ & $\begin{array}{l}152.1036(152.1030,3.94), \mathbf{1 3 5 . 0 7 6 9}(135.0765,2.96), \\
107.0819(107.0816,2.80), 77.0712(77.0710,2.60)\end{array}$ \\
\hline ТP13 & 6.41 & $\begin{array}{l}140.0570 \\
(140.0567,2.14) \\
\text { EC: } \mathrm{C}_{4} \mathrm{H}_{6} \mathrm{~N}_{5} \mathrm{O}^{+} \\
\end{array}$ & $\begin{array}{l}123.0305(123.0302,2.44), 97.0510 \quad(97.0509,1.03), \\
95.0356(95.0353,3.16) 86.0346(86.0349,-3.49), \mathbf{6 8 . 0 2 4 6} \\
(68.0244,2.94)\end{array}$ \\
\hline ТP14 & 6.57 & $\begin{array}{l}\mathbf{2 6 9 . 1 0 1 7} \\
(269.1017,3.72) \\
\text { EC: } \mathrm{C}_{10} \mathrm{H}_{9} \mathrm{~N}_{10}{ }^{+}\end{array}$ & $\begin{array}{lllll}252.0748 & (252.0741,2.78), & 242.0903 & (242.0898, & 2.07), \\
227.0794 & (227.0789,2.20), & 174.0528 & (174.0523, & 2.87), \\
161.0576 & (161.0571,3.10), 81.0199(81.0196,3.70) & \end{array}$ \\
\hline
\end{tabular}




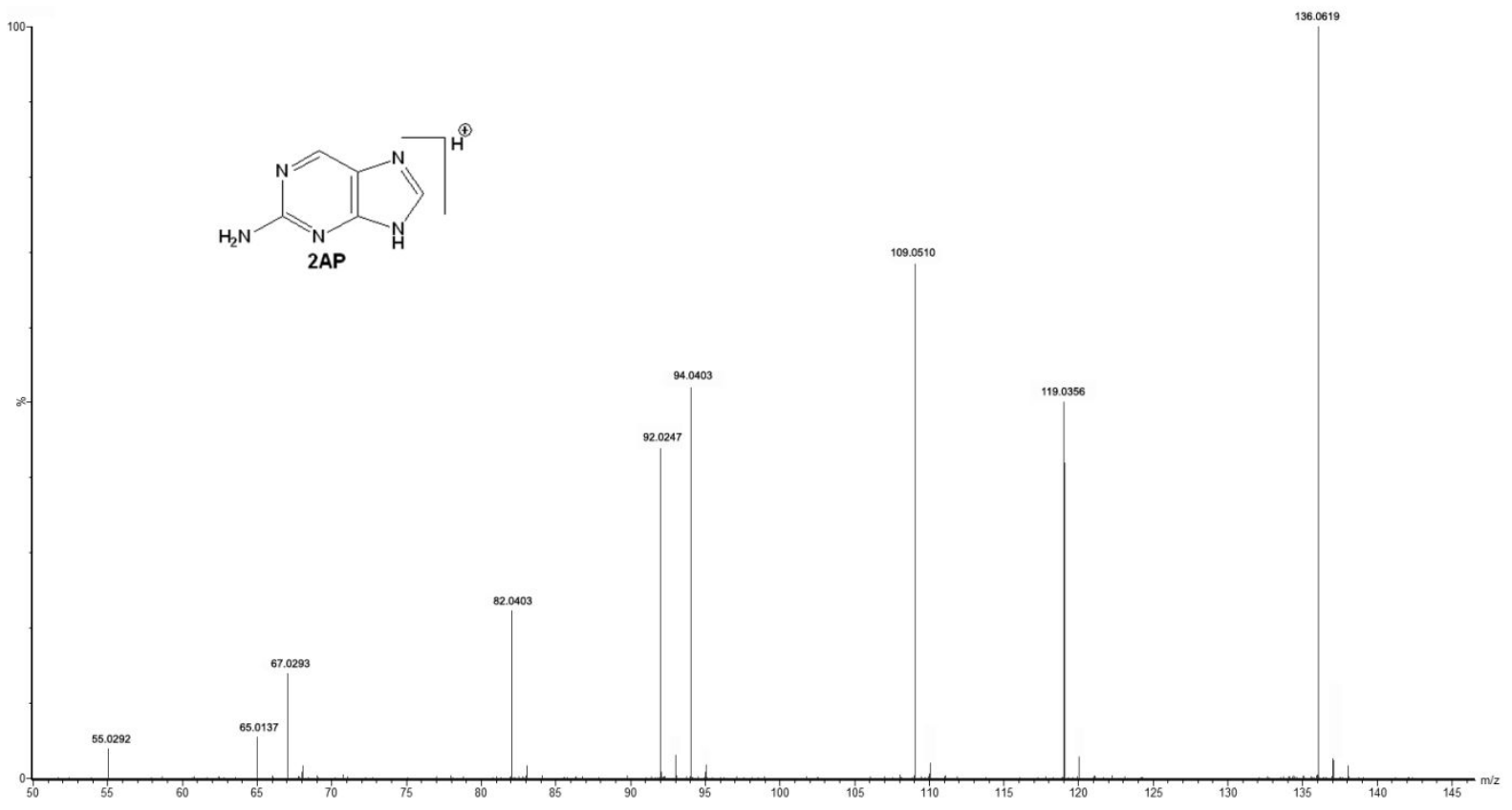

Figure S2 CID induced mass spectrum of 2AP

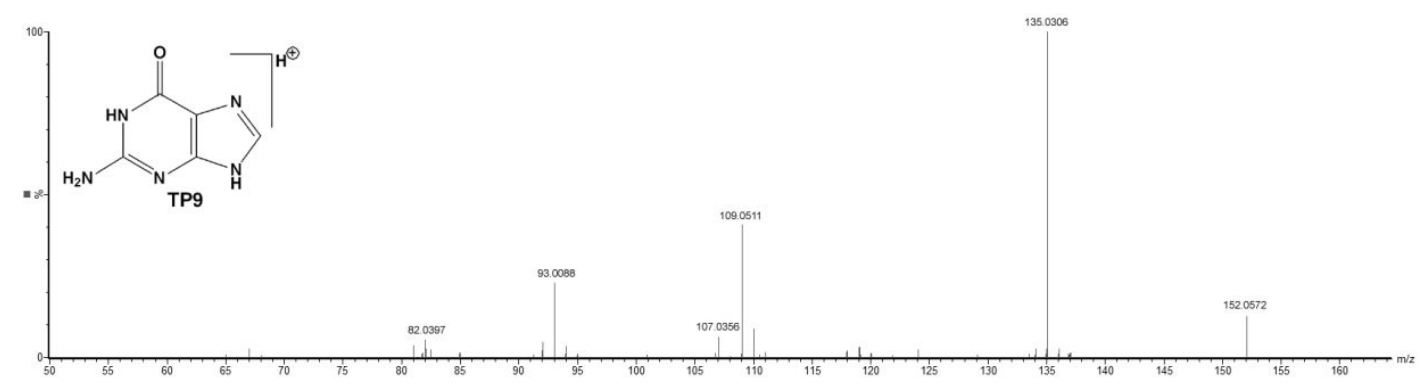

Figure S3 CID induced mass spectrum of TP9

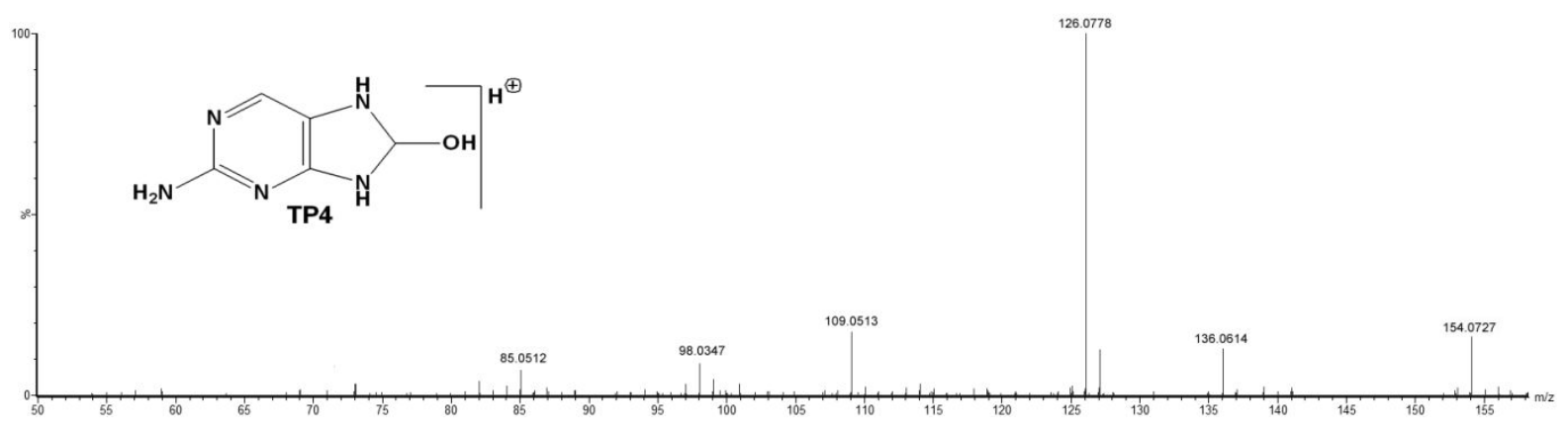

Figure S4 CID induced mass spectrum of TP4 


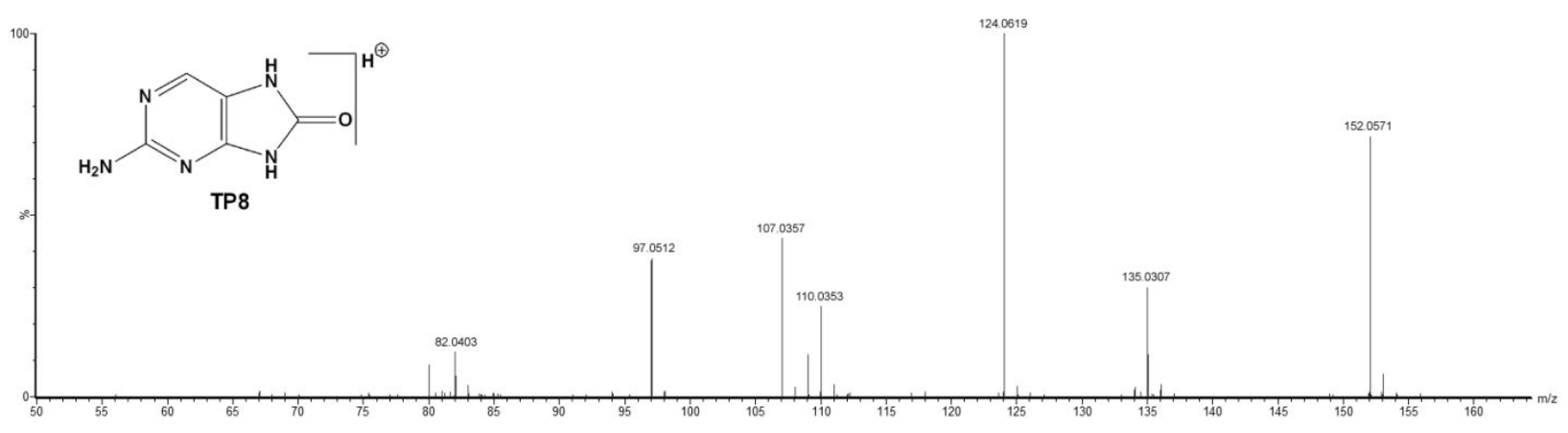

Figure S5 CID induced mass spectrum of TP8

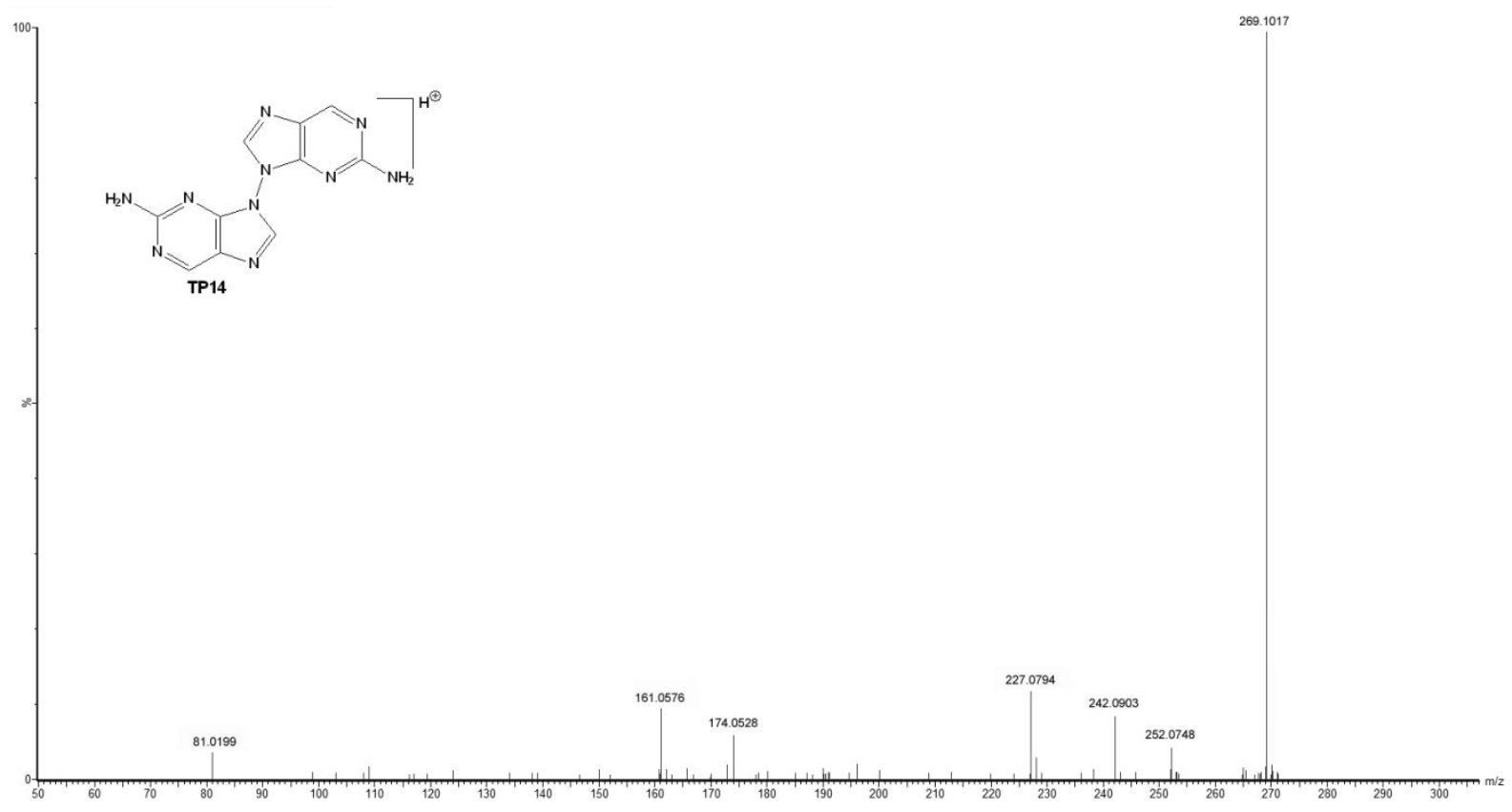

Figure S6 CID induced mass spectrum of TP14 
Scheme S1 Fragmentation pattern of TP1

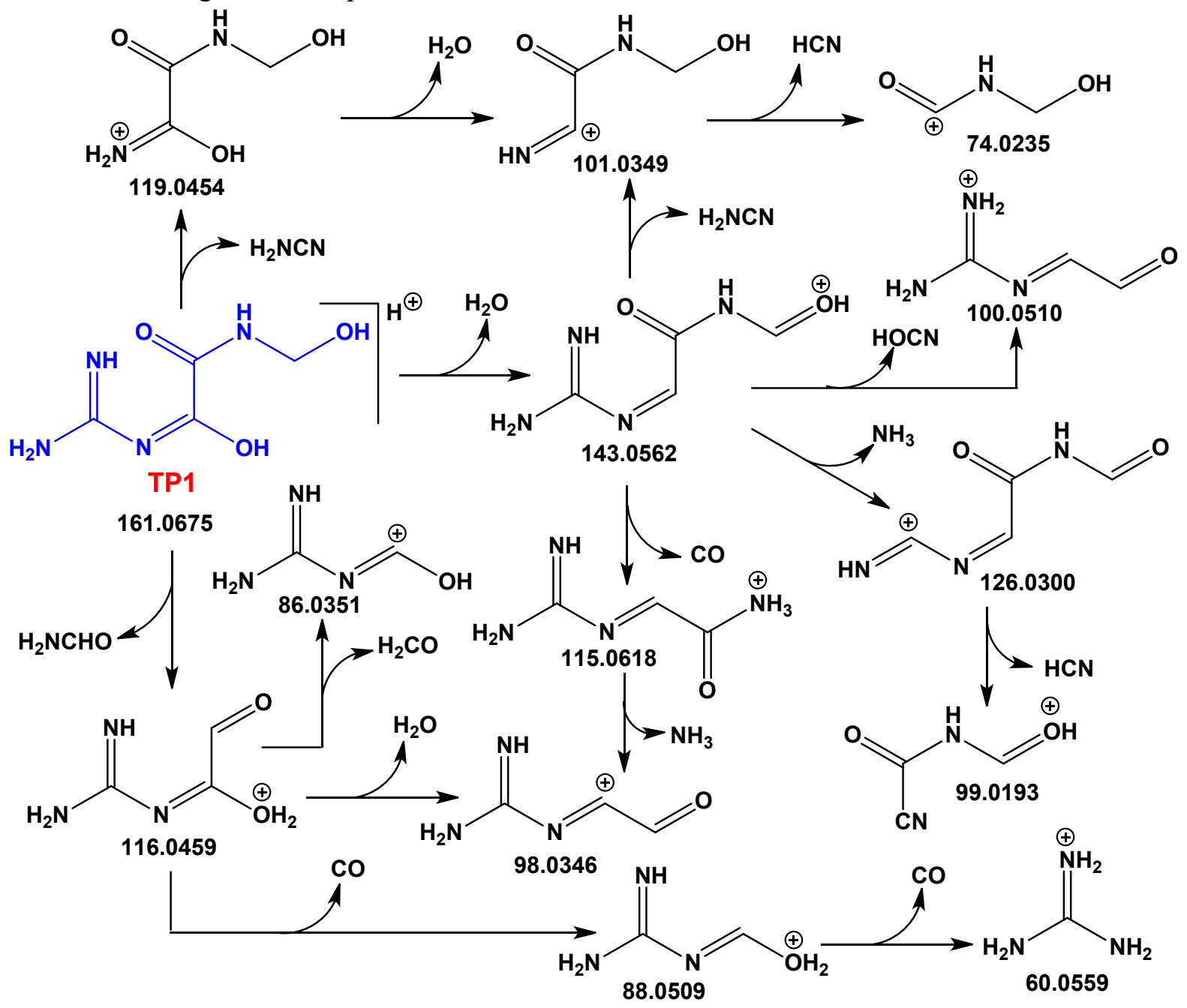


Scheme S2 Fragmentation pattern of TP2

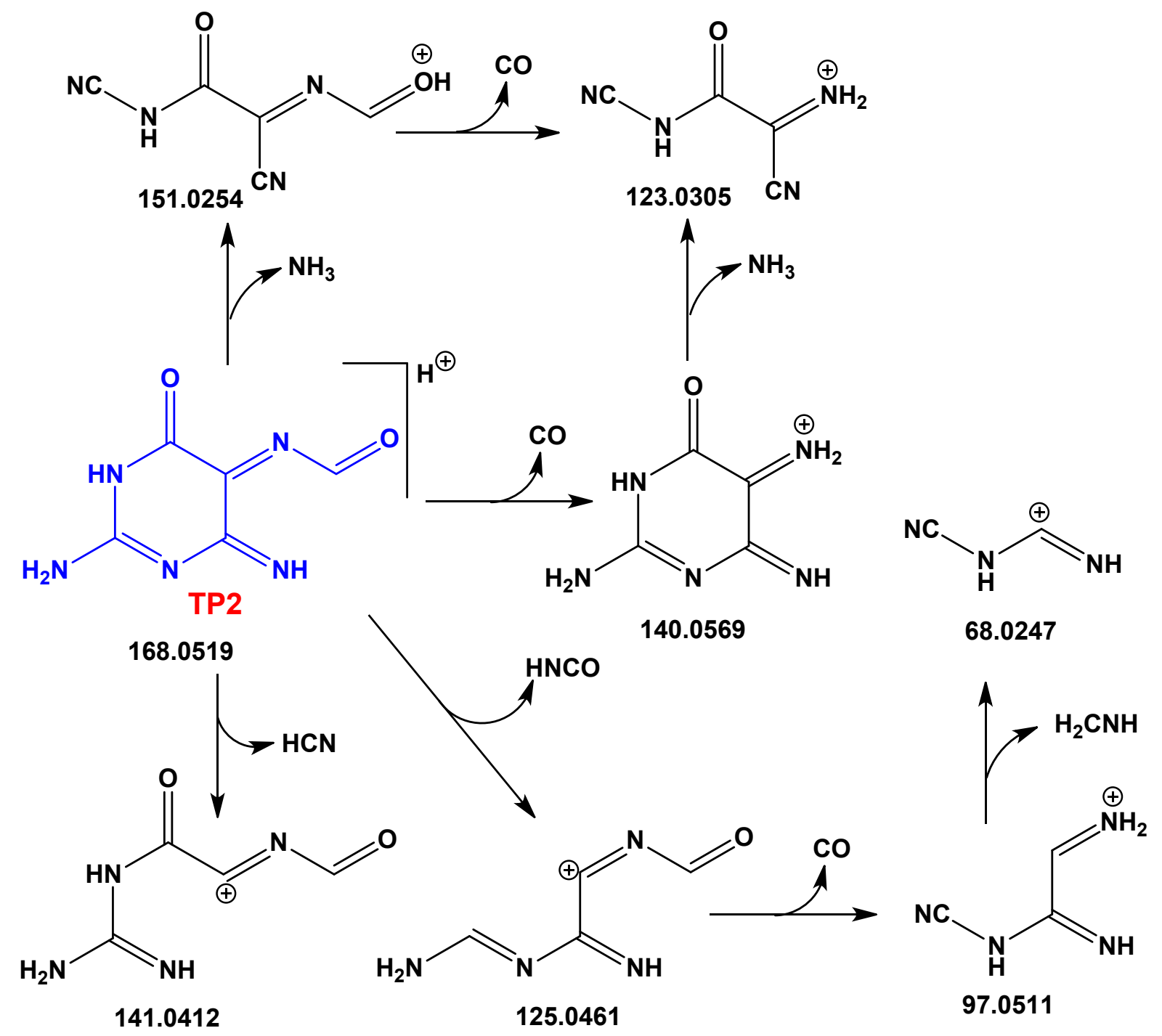


Scheme S3 Fragmentation pattern of TP3

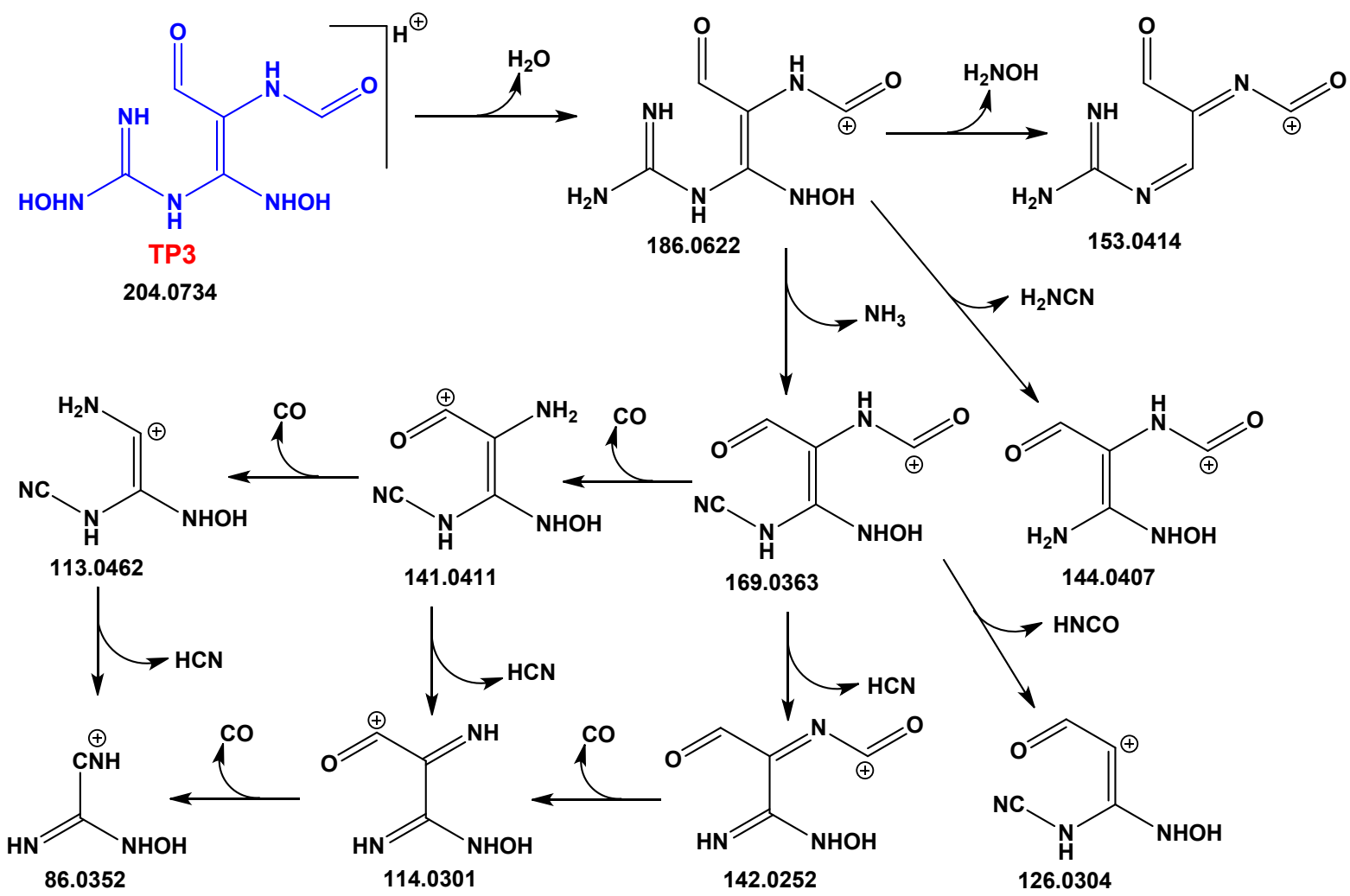


Scheme S4 Fragmentation pattern of TP5<smiles>CC1CC1NOn1[nH]c(=O)[nH]c1=O</smiles><smiles>N#CC(=O)NC=O</smiles><smiles>N#CN=CN</smiles>

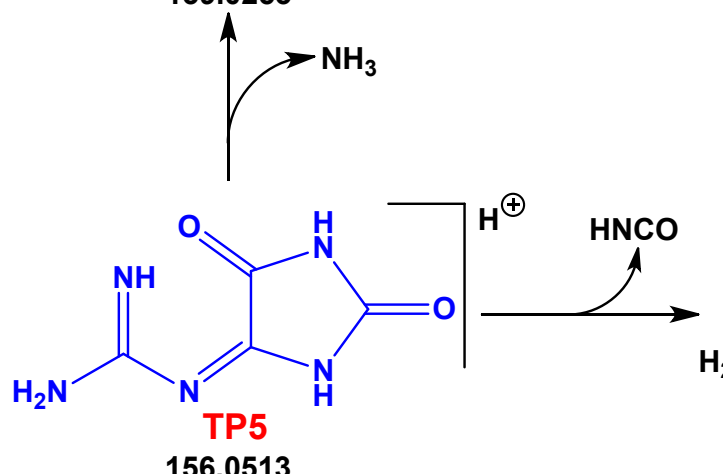

97.0035

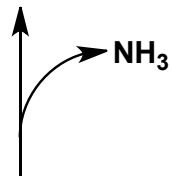

.

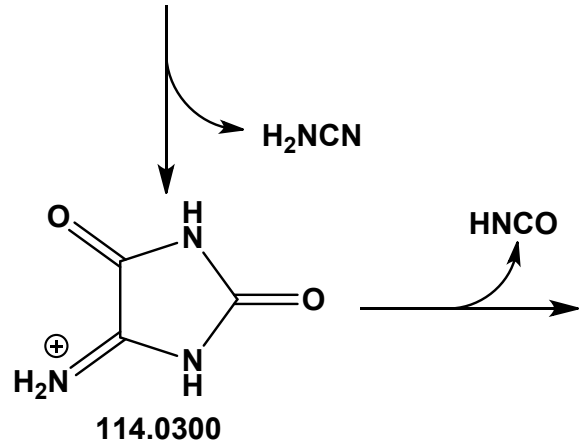<smiles>N=CNC=O</smiles>

114.0300 71.0243 
Scheme S5 Fragmentation pattern of TP6<smiles>CC1C(=O)C1C(=N)N=C(C=O)C(=N)O</smiles>

114.0300<smiles>CC1CC1CNN</smiles>

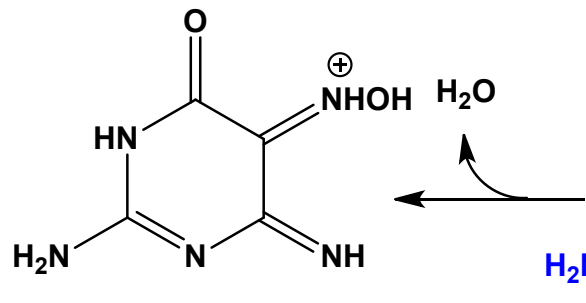

156.0513

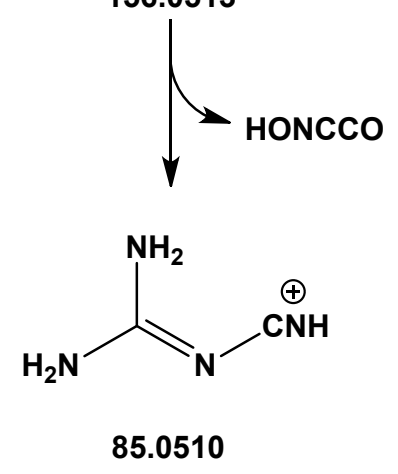

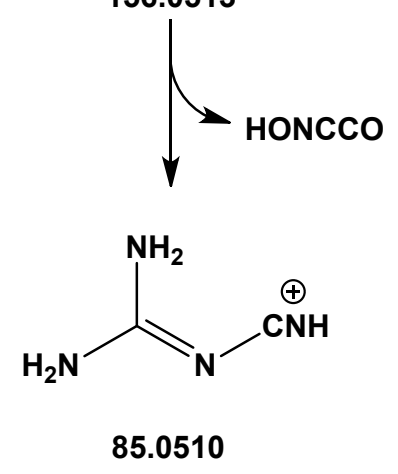

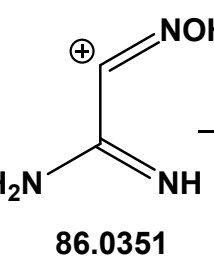<smiles>CC1C(N)[C@@H]1NC=N</smiles>

69.0087<smiles>N#CN=CC(C=O)=NO</smiles><smiles>CC1C(C)C1N</smiles>
$\mathrm{H}_{2} \mathrm{~N}$<smiles>CC[C@H](O)CNC(N)=O</smiles><smiles>CC1C[C@@H]1NO</smiles><smiles>N=C(C=O)/C=N\C(=N)N</smiles>

TP6

$\mathrm{NHOH}$

174.0625<smiles>CC1C(C)C1NOO</smiles><smiles>CC1CC1C(C)C(N)=NO</smiles>

99.0193 
Scheme S6 Fragmentation pattern of TP7<smiles>NC(=O)N[C+]=O</smiles>

87.0193<smiles>CC1C(C)C1N</smiles><smiles>O=C=N[C+]=O</smiles>

69.9926<smiles>CCCCC(C)C=O</smiles><smiles>C1CC[Co]CC1</smiles>

0

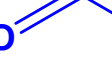<smiles>[1H]</smiles>

TP7

129.0300

Scheme S7 Fragmentation pattern of TP10<smiles>N#CC=CNC#N</smiles>

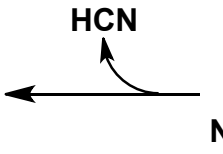<smiles>N#CN=C[C+]=CNC#N</smiles>

119.0349<smiles>C#CN=CN</smiles>

67.0293

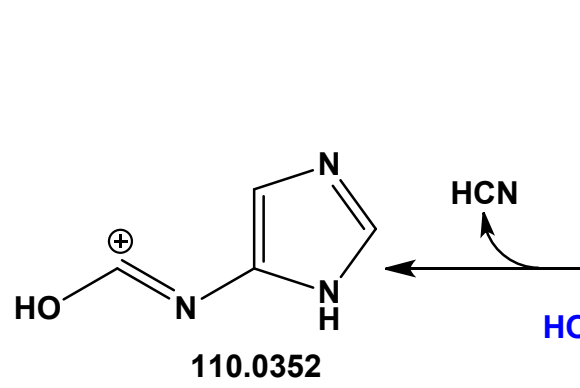<smiles>CC(C)C(=O)O</smiles><smiles>N=C1C=NC=N1</smiles><smiles>CC1C[C@@H](NC(N)=O)[C@H]1C(N)=O</smiles>

86.0236<smiles>O=C=C[C+]=O</smiles>

68.9974<smiles>CC1C(C)C1N</smiles><smiles>CC1C(C)C1C=O</smiles><smiles>[18OH]</smiles><smiles>Cc1ncc2nc[nH]c2n1</smiles><smiles>CC1C(C#N)[C@@H]1C(=O)c1c[nH]cn1</smiles>

94.0397<smiles>[Y]C(C)[C@@H](C)NC</smiles>

$\stackrel{\oplus}{\mathrm{NN}}$ 53.0137 
Scheme 8 Fragmentation pattern of TP11
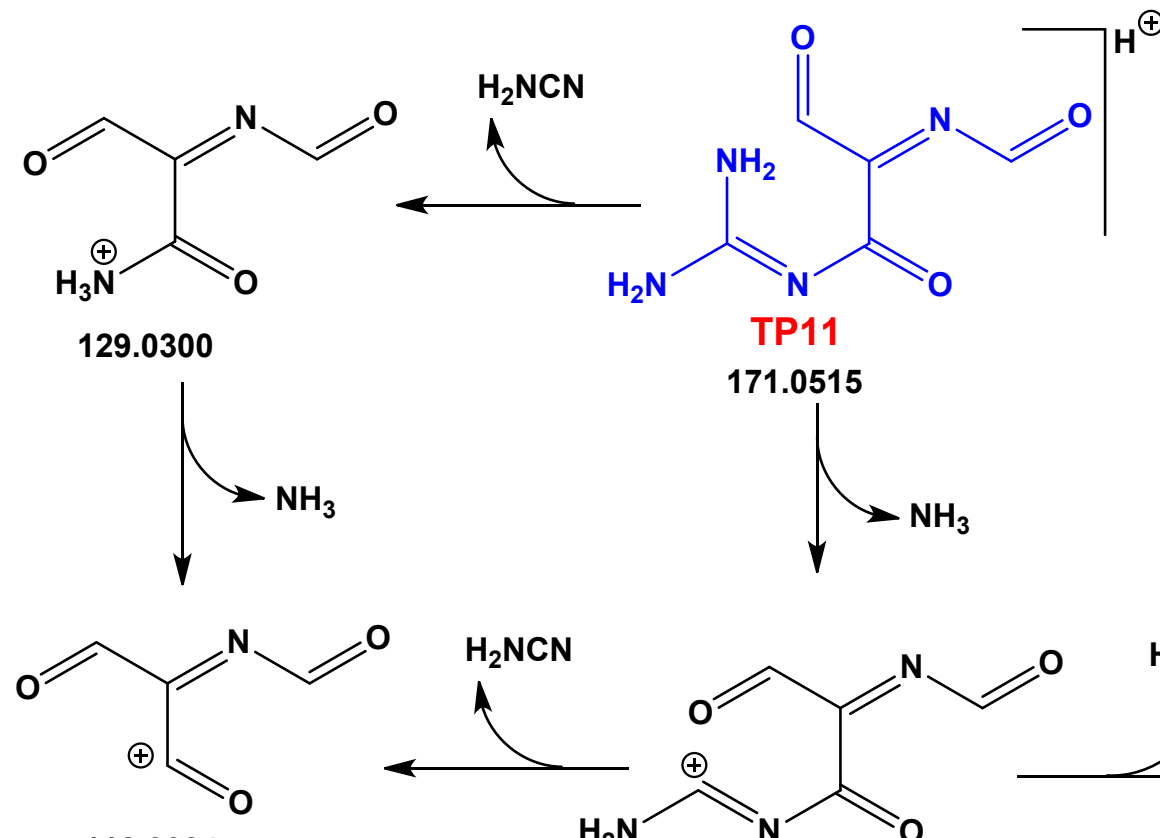

112.0034<smiles>CC1C(=O)C1C=O</smiles>

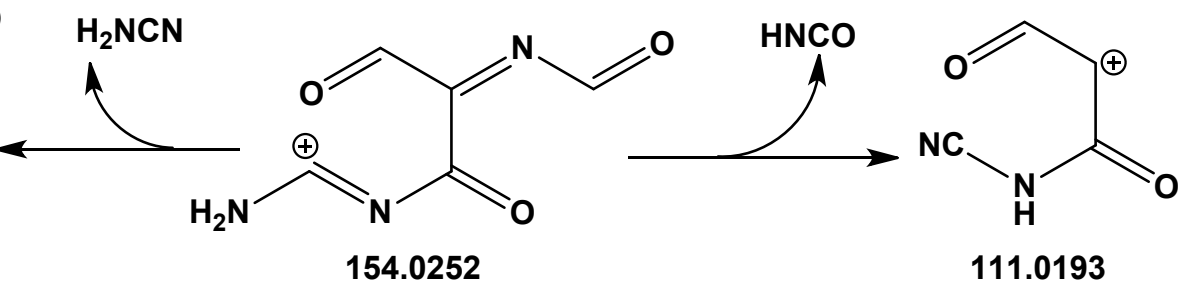

84.0083 
Scheme S9 Fragmentation pattern of TP12

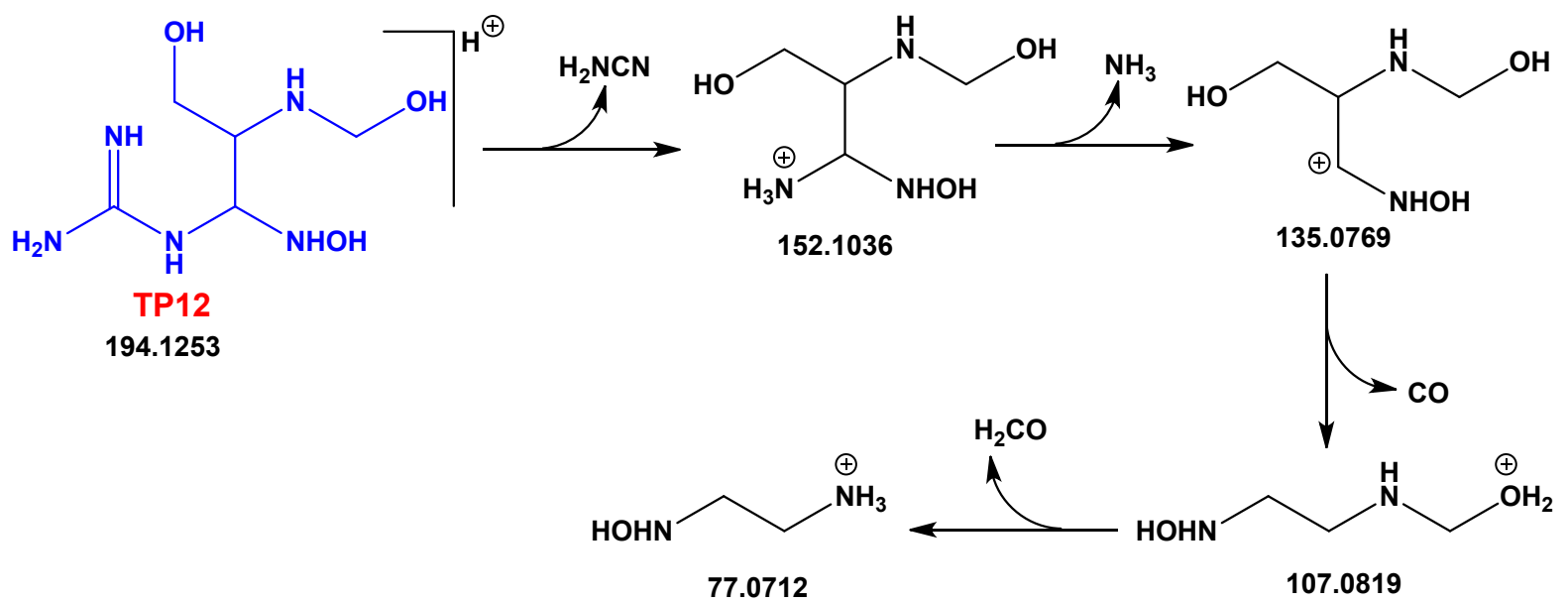

Scheme S10 Fragmentation pattern of TP13<smiles></smiles>

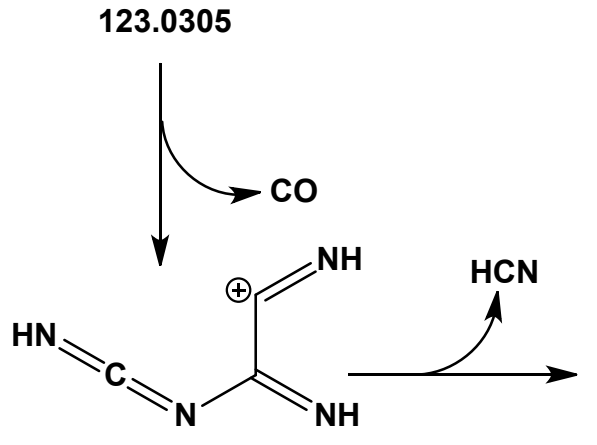
95.0356<smiles>CCC(C)(C)C(=N)N=C(N)NC(C)(C)C</smiles>
$\mathbf{H}^{\oplus}$

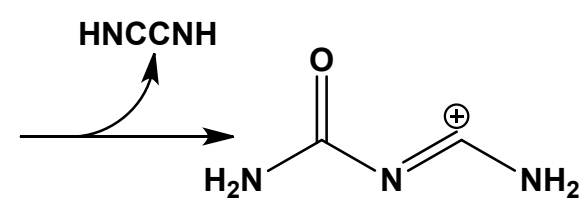
86.0346

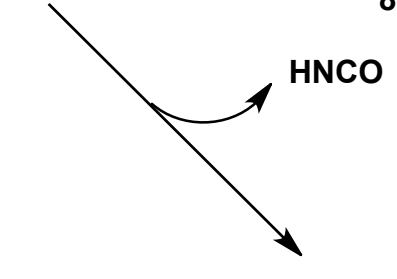<smiles>N#CNC=N</smiles>

68.0246<smiles>CC(C)C(C)C</smiles><smiles>N=C(CCCCCN)N=CN</smiles>

97.0510 